\title{
Efeitos da condição sexual de cordeiros machos Texel x Corriedale na produção e qualidade da lã
}

Tavares Costa, P.@; Amarilho Silveira, F.; Tavares Costa, R.; Duarte Farias, G.; Albandes Fernandes, T.; Souza Mendonça, F.; Zambarda Vaz, R. e de Mendonça, G.

Universidade Federal de Pelotas. Brasil.

\section{RESUMO}

\section{PalaVRas chaVe adicionaIS}

Ovinos.

Castração.

Criptorquidismo.

Fibra de lã.

Peso de velo.
O estudo foi realizado com objetivo de avaliar o efeito da condição sexual de cordeiros machos cruza Texel $x$ Corriedale mantidos sem a castração, castrados ou induzidos ao criptorquidismo na produção e qualidade da lã. Foram utilizados 49 animais cruza Texel $\times$ Corriedale, criados na região do Bioma Pampa, no Estado do Rio Grande do Sul, em condições extensivas de pastagem natural, divididos em três grupos, conforme a condição sexual: não castrados $(n=16)$, castrados $(n=17)$ ou induzidos ao criptorquidismo $(n=16)$, sendo tosquiados com idade média de 160 dias. Foi utilizada a análise de variância para avaliar o efeito da condição sexual. $O$ crescimento e o comprimento da fibra de lã não diferiram significativamente $(P>0,05)$ entre os grupos. $O$ peso de velo sujo de cordeiros castrados e inteiros não diferiu entre si $(P>0,05)$, sendo ambos superiores $(P<0,05)$ aos animais criptorquidas induzidos. Não houveram diferenças $(P>0,05)$ para as características qualitativas de diâmetro médio da fibra de lã, coeficiente de variação do diâmetro, pontos mais finos e mais grossos ao longo da fibra. Conclui-se que a condição sexual não afeta as características qualitativas da lã. Cordeiros induzidos ao criptorquidismo apresentam peso de velo sujo inferior aos demais.

\section{Effects of the Sexual condition of Texel $x$ Corriedale male lambs on wool production and quality}

\section{SUMMARY}

\section{ADDITIONAL KEYWORDS}

\section{Sheep.}

Castration.

Cryptorchidism.

Wool fiber.

Fleece weight.

\section{INFORMATION}

\section{Cronología del artículo.}

Recibido/Received: 21.12.2016

Aceptado/Accepted: 07.05.2018

On-line: 15.07 .2018

Correspondencia a los autores/Contact e-mail:

pablocostta@hotmail.com
The study was conducted to evaluate the effects of male lambs sexual condition on production and quality of wool. The lambs were kept under extensive system in natural pasture of the Pampa biome in Rio Grande do Sul State. The experiment was carried out with forty-nine male lambs, crossbred Corriedale $x$ Texel. Animals were divided into three groups according to sexual condition: non-castrated ( $n=16)$, castrated ( $n=17)$ or cryptorchidism-induced ( $n=16)$. Lambs were sheared with 160 days of age. The analysis of variance was used as statistical test to evaluate the effect of sexual condition. The growth and length of wool fiber did not differ significantly between groups $(P>0.05)$. The dirty fleece weight was not different between non-castrated and castrated male lambs ( $P>0.05)$, however both groups showed higher weight of dirty fleece compared with the cryptorchidism-induced group $(\mathrm{P}<0.05)$. There were no significant differences between groups regarding to the wool qualitative characteristics as fiber diameter average and coefficient of variation, and proportion of finer and coarser points along the wool fiber $(\mathrm{P}>0.05)$. It is concluded that the sexual condition of male lambs does not affect the wool qualitative characteristics. Cryptorchidism-induced lambs has lower dirty fleece weight than other groups.

\section{INTRODUÇÃO}

O Brasil possui um rebanho ovino de aproximadamente 18,41 milhões de cabeças, estando 21,5\% concentrados no Rio Grande do Sul, com o maior efetivo de animais e constituindo o maior produtor de lã e carne ovina do País (IBGE, 2016). O Estado é responsável por $68 \%$ da produção brasileira de carne ovina (Ávila et al.
2013, p. 2423) e detêm $91,9 \%$ da produção nacional de lã (IBGE, 2016). O rebanho ovino da região é formado, em sua maioria, pelas raças consideradas laneiras e/ ou de dupla aptidão, das quais a Corriedale é a mais numerosa (Feijó et al. 2017, p. 98). Com a valorização da carne ovina, os produtores têm introduzido raças com maior aptidão para sua produção, sendo a raça Texel a mais utilizada em cruzamentos. A utilização 
de cruzamento de ovelhas adaptadas a uma região, como é o caso da raça Corriedale no Rio Grande do Sul, com raças paternas especializadas para carne é uma alternativa para aumentar a eficiência dos sistemas produtivos (Osório et al. 2002, p. 1469).

Em um sistema produtivo de raças de dupla aptidão, a carne ovina participa, em média, com $54 \%$ da receita bruta total da atividade, enquanto o produto lã com $46 \%$ (Viana \& Silveira 2009, p. 1189). Estes dados demonstram que, embora a valorização da carne, a lã continua tendo grande importância no sistema produtivo de raças mistas, gerando receitas significativas e contribuindo para os resultados econômicos positivos da exploração.

No que se refere a produção de lã, o crescimento das fibras é marcadamente hormônio-dependente, onde a manipulação experimental do status hormonal provoca grandes alterações na taxa de crescimento (Amarilho-Silveira et al. 2015, p. 18). Conforme Wallace (2000, p. 261), os hormônios hipofisários exercem uma influência controladora sobre o crescimento de lã. Khan et al. (2012, p. 13764) descrevem que o crescimento da lã em ovinos é influenciado pelo hormônio do crescimento. É consenso entre os estudiosos que, na idade adulta, a produção de lã de machos inteiros é superior à de castrados (Minola \& Elissondo 1990, p. 20; Amarilho-Silveira 2015, p. 19), característica essa decorrente do maior tamanho corporal e peso vivo adulto dos animais não castrados, e influenciada por uma adequada atividade testicular e um bom equilíbrio endócrino (Minola \& Elissondo 1990, p. 20).

Em relação ao processo de comercialização, a qualidade da lã é cada vez mais valorizada pelos mercados mais exigentes, que se baseiam nos aspectos de medição objetiva, indicadores de rendimento e processamento industrial (Peña et al. 2016, p. 14; Amarilho-Silveira et al. 2017, pp. 137-138; Gómez et al. 2017, p. 358).

Quando o foco é produzir animais jovens para o abate, até os 180 dias de vida, muitas vezes não se realiza a castração, com intuito de se evitar possíveis perdas advindas do procedimento. A tosquia de animais lanados é uma prática comum, sendo realizada anualmente, inclusive em cordeiros que serão enviados para o abate, possibilitando elevar o rendimento de carcaça e agregando valor a produção por meio da comercialização da lã. No entanto, existem poucos estudos que caracterizem a qualidade e a produção de lã em cordeiros, sendo mais escassas informações no tocante à influência da castração nestas, sendo necessário avaliar tais características, para melhor avaliar a produtividade do sistema como um todo. Dessa forma, o objetivo deste trabalho foi avaliar o efeito das condições sexuais de cordeiros machos cruza Texel $x$ Corriedale não castrados, castrados ou induzidos ao criptorquidismo na produção e qualidade da lã.

\section{MATERIAL E MÉTODOS}

A condução da pesquisa foi aprovada pela Comissão de Ética em Experimentação Animal da Universidade Federal de Pelotas, sob o código CEEA 3118.
O estudo foi desenvolvido em uma propriedade rural localizada no município de Pinheiro Machado, pertencente à região da Encosta do Sudeste, no sul do Estado do Rio Grande do Sul, localizada entre as coordenadas $31^{\circ} 31^{\prime} 30^{\prime \prime}$ latitude sul e $53^{\circ} 29^{\prime} 45^{\prime \prime}$ longitude oeste, nos campos do Bioma Pampa. A altitude, temperatura e precipitação pluviométrica médias anuais são de 365 metros, $16^{\circ} \mathrm{C}$ e $1.380 \mathrm{~mm}$, respectivamente, e o clima é temperado (IBGE, 2016).

Foram utilizados 49 cordeiros machos provenientes do cruzamento entre matrizes da raça Corriedale com reprodutores da raça Texel, divididos em três grupos, conforme a condição sexual: induzidos ao criptorquidismo $(\mathrm{n}=16)$, não castrados $(\mathrm{n}=16)$ ou castrados $(\mathrm{n}=17)$.Os cordeiros foram identificados individualmente ao nascimento, através da aplicação de brincos numerados específicos para a espécie, e mantidos em sistema de manejo extensivo, permanecendo juntamente às mães em piquetes de pastagem natural, com fontes naturais de água e sombra. Os animais foram castrados ou induzidos ao criptorquidismo quando atingiram idade média de 35 dias. A castração foi realizada conforme procedimento cirúrgico descrito por Turner \& Mcilwraith (2002, pp. 263-264). A indução ao criptorquidismo foi realizada mediante utilização de anéis de borracha, específicos para esse fim, colocados na base da bolsa escrotal com aplicador específico, após reintrodução manual dos testículos na cavidade abdominal.

Os cordeiros foram preventivamente vacinados para ectima contagioso aos 35 dias de vida. A infestação endoparasitária foi monitorada por meio de exames de contagem do número de ovos por grama de fezes (OPG) pela técnica de McMaster, desenvolvida por Gordon \& Whitlock (1939, p. 51), sendo realizadas dosificações sempre que a média superou 500 OPGs. Os animais foram acompanhados constantemente durante o experimento e tomadas medidas sanitárias curativas sempre que necessário.

A idade média no momento das primeiras mensurações das características de lã era de 91 dias. Para verificar o comprimento da fibra da lã e o seu crescimento, os cordeiros eram examinados individualmente, em estação, estando contidos manualmente. As medições foram realizadas na região do costilhar direito dos cordeiros, com a utilização de paquímetro analógico. As avaliações foram realizadas aos 91 dias de idade e no momento da tosquia dos animais, sendo, as mesmas, durante o intervalo entre estas medidas, avaliadas a cada 28 dias.

O crescimento da fibra foi determinado em $\mathrm{mm} /$ dia, e calculado através da fórmula: $\mathrm{CR}=$ (comprimento da fibra de lã na tosquia - comprimento da fibra de lã aos 91 dias)/número de dias entre as medições.

A tosquia foi realizada quando os cordeiros apresentaram a idade média de 160 dias. Após a mesma, os velos foram pesados em balança digital (balança com capacidade de $50 \mathrm{~kg}$ e precisão de $10 \mathrm{~g}$, marca WeiHeng ${ }^{\circledR}$ ) para obtenção do peso de velo sujo (PVS), em $\mathrm{kg}$. 
Momentos antes da tosquia foram coletadas amostras de lã $( \pm 50 \mathrm{~g})$ da região do costilhar direito de cada animal. As amostras foram identificadas individualmente, embaladas em sacos plásticos e acondicionadas em caixa térmica. Após, foram encaminhadas ao laboratório de medidas objetivas Micralan, Montevidéu, Uruguai, para análise das características qualitativas, sendo submetidas às análises objetivas provenientes do Optical Fibre Diameter Analysis (OFDA 2000 ${ }^{\circledR}$ ), as quais consistem na aferição do diâmetro médio da fibra de lã (DF), coeficiente de variação do diâmetro(CV), e pontos mais finos (PF) e mais grossos (PG) ao longo da fibra (Baxter 2001, pp. 119-130).

Após a tosquia, respeitando um período de jejum alimentar e hídrico de 12 horas, foram estimados o escore de condição corporal (ECC) e realizadas as pesagens individuais dos animais. O método de avaliação da condição corporal usado foi o descrito por Osório et al. (2008, pp. 113-116). Os pesos corporais individuais foram obtidos com balança digital (WeiHeng ${ }^{\circledR}$ ), em kg.

Os dados foram submetidos a análise de variância para avaliar o efeito da condição sexual e as médias foram comparadas pelo teste F. Quando verificada significância estatística pela análise de variância, as médias foram contrastadas pelo teste de DMS Fisher. As análises foram realizadas com o auxílio do programa estatístico BioEstat 5.3 (Ayres et al. 2007), ao nível de significância de 5\%.

\section{RESULTADOS E DISCUSSÃO}

O peso vivo e o ECC não diferiram entre os grupos (P>0,05 - Tabela I). Crouse et al. (1981, p. 381), afirmam que é de se esperar uma melhor performance de machos não castrados em relação aos castrados, mas principalmente quando é proporcionado um alto nível de alimentação aos cordeiros, o que não foi oportunizado no presente trabalho, em virtude dos animais terem sido mantidos em sistema extensivo de criação, sobre campos de pastagens nativas, que normalmente apresentam baixas disponibilidades de alimento de qualidade, o que pode ter impossibilitado a observação de diferenças no peso vivo e na deposição de tecido adiposo.

O peso de velo sujo não diferiu entre cordeiros castrados e não castrados $(\mathrm{P}>0,05)$, tendo ambos pesos superiores $(\mathrm{P}<0,05)$ ao observado nos cordeiros induzidos ao criptorquidismo (Tabela I).

Resultados diferentes foram encontrados por Hopkins et al. (1992, p. 445), que estudando borregos Merino, Ideal e Merino x Ideal castrados e submetidos ao criptorquidismo induzido, constataram PVS superior nos criptorquidas em relação aos castrados, de, respectivamente, $4,3 \mathrm{~kg} \mathrm{e} \mathrm{4,0} \mathrm{kg,} \mathrm{aos} 365$ dias de idade.

$\mathrm{O}$ peso de velo sujo mensurado em animais castrados e inteiros foi semelhante ao obtido por Oliveira \& Figueiró (1979, p. 262), em cordeiros da raça Corriedale, nascidos na mesma época do ano e tosquiados com idade semelhante. Minola \& Elissondo (1990, p. 20) taxam aos machos inteiros uma maior produção de lã quando comparados aos castrados. No entanto, tal comparação é inferida comparando animais já em idade adulta, sendo essa diferença mais relacionada ao tamanho corporal distinto entre animais inteiros e castrados do que propriamente à condição sexual.

$\mathrm{O}$ crescimento da fibra de lã não diferiu significativamente $(P>0,05)$ entre os grupos. Tal característica, por ser hormônio-dependente, está mais relacionada aos hormônios hipofisários, do que propriamente com a testosterona (Wallace 2000, p. 261). A prova disso é a manipulação experimental descrita por Osório et al. (2014, p. 454), em que uma vez extirpada a glândula pituitária, o crescimento da lã cessa ou é interrompido, visto que, a administração do hormônio estimulador da tireoide (TSH) pode restabelecer o crescimento em ovinos hipofisectomisados.

O comprimento da fibra de lã não diferiu $(\mathrm{P}>0,05)$ entre os grupos em nenhum dos períodos analisados, evidenciando que a realização da castração e o tipo de castração utilizado não influenciam o crescimento das fibras de lã.

Segundo Butler (1982, p. 311), um fator que influencia quantitativamente e qualitativamente a produção de fibra de lã em ovinos é o número de folículos secundários, o que afeta a densidade do velo. Possivelmente, cordeiros criptorquidas induzidos apresentaram peso de velo sujo inferior aos demais devido a um menor número de folículos secundários de lã, e, consequentemente, menor densidade do velo, visto que apresentaram peso vivo, escore de condição corporal e

Tabela I. Médias e erro padrão para as características escore de condição corporal (ECC), peso vivo (PV), peso de velo sujo (PVS), crescimento da fibra de lã (CR) e comprimento de fibra de lã (CF) de cordeiros castrados, não castrados ou induzidos ao criptorquidismo (Average and standard error for the characteristics of the body condition score (ECC), live Weight (PV), Dirty Fleece Weight (PVS), Growth of wool fiber (CR) and length of wool fiber (CF) of castrated lambs, not castrated or induced to cryptorchidism).

\begin{tabular}{|c|c|c|c|}
\hline \multirow{2}{*}{ Parâmetro } & \multicolumn{3}{|c|}{ Condição Sexual } \\
\hline & Criptorquidas & Não castrados & Castrados \\
\hline ECC (1 a 5) & $2,28 \pm 0,16^{a}$ & $2,50 \pm 0,16^{a}$ & $2,68 \pm 0,12^{\mathrm{a}}$ \\
\hline $\mathrm{PV}(\mathrm{kg})$ & $27,69 \pm 1,20^{a}$ & $31,00 \pm 1,37^{a}$ & $30,31 \pm 0,98^{a}$ \\
\hline PVS (kg) & $1,144 \pm 0,19^{b}$ & $1,407 \pm 0,27^{a}$ & $1.454 \pm 0,19^{a}$ \\
\hline CR (mm/dia) & $0,268 \pm 0,09^{a}$ & $0,228 \pm 0,08^{a}$ & $0.261 \pm 0,08^{a}$ \\
\hline $\mathrm{CF}(\mathrm{mm})$ & $51,7 \pm 10,7^{a}$ & $52,5 \pm 9,9^{a}$ & $54.7 \pm 10,5^{a}$ \\
\hline
\end{tabular}

*Médias na mesma linha seguidas de letras distintas diferem entre si a $5 \%$ de significância. 
Tabela II. Médias e erro padrão para o diâmetro médio da fibra de lã (DF), coeficiente de variação do diâmetro (CV), e pontos mais finos (PF) e mais grossos (PG) ao longo da fibra de cordeiros castrados, não castrados ou induzidos ao criptorquidismo (Average and standard error for the average diameter of wool fiber (DF), coefficient of variation of diameter (CV), and Finer (PF) and thicker (PG) points along the fibre of castrated lambs, not castrated or induced to cryptorchidism).

\begin{tabular}{|c|c|c|c|}
\hline \multirow{2}{*}{ Parâmetro } & \multicolumn{3}{|c|}{ Condição Sexual } \\
\hline & Criptorquidas & Não castrados & Castrados \\
\hline $\mathrm{DF}(\mu \mathrm{m})$ & $26,66 \pm 0,33^{a}$ & $26,63 \pm 0,63^{a}$ & $27,18 \pm 0,48^{a}$ \\
\hline CV (\%) & $23,84 \pm 0,78^{a}$ & $24,38 \pm 0,62^{\mathrm{a}}$ & $23,20 \pm 0,72^{a}$ \\
\hline $\mathrm{PF}(\mu \mathrm{m})$ & $24,52 \pm 0,39^{a}$ & $25,01 \pm 0,65^{\mathrm{a}}$ & $25,6 \pm 0,51^{a}$ \\
\hline$P G(\mu \mathrm{m})$ & $29,24 \pm 0,35^{a}$ & $28,77 \pm 0,72^{a}$ & $29,49 \pm 0,49^{a}$ \\
\hline
\end{tabular}

*As médias não diferiram entre os grupos em nenhuma das características estudadas, a $5 \%$ de significância.

crescimento e comprimento da fibra de lã semelhante aos demais grupos.

Não foi verificada diferença significativa para o diâmetro médio da fibra de lã (P>0,05 - Tabela II). O que corrobora com Hopkins et al. (1992, p. 445), que não encontraram distinção entre o diâmetro médio das fibras de lã entre borregos Merino, Ideal e Merino x Ideal castrados ou submetidos ao criptorquidismo induzido. Foster et al. (1997, p. 303), em estudo semelhante, trabalhando com animais castrados, hemi-castrados (remoção de um dos testículos) ou criptorquidas induzidos da raça Merino, também não verificaram diferença entre o diâmetro médio das fibras de lã. No entanto, animais hemi-castrados produziram maior quantidade de lã, aferido pelo peso de velo limpo.

As demais caraterísticas qualitativas, como o coeficiente de variação do diâmetro, os pontos mais finos e os pontos mais grossos ao longo da fibra também não diferiram $(P>0,05)$ entre os grupos estudados (Tabela II).

De acordo com Sacchero et al. (2010, p. 40), em um grupo racial de um mesmo rebanho, as variações das características de diâmetro são muito mais dependentes do manejo nutricional, do que propriamente de um mérito individual, assim, afetando globalmente todo os indivíduos deste rebanho.

É importante ressaltar que a influência ambiental tem maior preponderância em animais com baixa variação genética para as características que não sofrem pressão de seleção, como é o caso dos cordeiros deste estudo, pois segundo Cardellino (1983, p. 13), mesmo em animais de uma mesma raça, se sofrerem determinado tipo de seleção ao longo dos anos, apresentam aspectos distintos acerca da qualidade da lã quando comparados a animais não selecionados. Tal fato ocorre, pois, mesmo que se selecione animais para apenas uma característica, outras acabam sendo afetadas concomitantemente.

No presente estudo, as matrizes do rebanho haviam sido selecionadas por várias gerações sucessivas para maior peso vivo e habilidade materna, não se despendendo maiores atenções às características de qualidade de lã, e, da parte paterna, utilizou-se apenas dois carneiros na estação reprodutiva, portanto, os cordeiros apresentavam características semelhantes, com baixa variabilidade genética, e, como foram submetidos as mesmas condições ambientais, não se observaram diferenças nas características qualitativas da lã.

\section{CONCLUSÕES}

Conclui-se que a condição sexual não afeta as características qualitativas da lã. Cordeiros induzidos ao criptorquidismo apresentam peso de velo sujo inferior ao de animais castrados e não castrados.

\section{BIBLIOGRAFIA}

Amarilho-Silveira, F, Brondani, WC \& Lemes, JS 2015, 'Lã: Características e fatores de produção', Archivos de Zootecnia, v. 64, no. R, 13-24. Amarilho-Silveira, F, Vicente, SV, Lemes, JS, Del Pino, FAB, Esteves, RM, Halfen, J, Tâmara, JQ, Da Vara, CC, Dionello, NJL \& Mattei, P 2017, 'Relação entre os níveis de metabólitos sanguíneos e a qualidade de lä', Archivos de Zootecnia, v. 66, no. 253, 137-140.

Ávila, VS, Fruet, APB, Barbieri, M, Bianchini, NH \& Dörr, AC 2013, 'O retorno da ovinocultura ao cenário produtivo do Rio Grande do Sul', Revista Eletrônica em Gestão, Educação e Tecnologia Ambiental, v. 11, no. 11, pp. 2419-2426.

Ayres, M, Junior, MA, Ayres, DL \& Santos, AAS 2007, BioEstat 5.3: Aplicações estatísticas nas áreas das Ciências Biomédicas, Sociedade Civil Mamirauá, Belém. 324p.

Baxter, P 2001, 'On-farm classing of animals and fleeces with the OFDA 2000', Wool Technologyand Sheep Breeding, v. 49, no. 2, pp. 119-137.

Butler, LG 1982, 'The effect of birth status on the level and efficiency of wool production by New Zealand Corriedale two-tooth rams and ewes', Animal Science, v. 35, no. 3, pp. 309-312.

Cardellino, R 1983, 'Importancia de las características de la lana', Secretariado Uruguayo de la Lana. Ovinos y Lanas, n. 10.

Crouse, JD, Busboom, JR, Field, RA \& Ferrell, CL 1981, 'The effects of breed, sex, location and slaughter weight on lamb growth carcass composition and meat flavour', Journal of Animal Science, v. 53, no. 2, p. 376-386.

Feijó, FD, Mendonça, G, Costa, PT, Costa, RT, Benedetti, M \& Machado, MC 2017, 'Onset of reproductive activity of white and natural colored corriedale ewe lambs', Acta Veterinaria Brasilica, v. 11, no. 2, pp. 98-103.

Foster, FM, Jackson, RB, Hopkins, DL \& Corkrey, R 1997, 'Production and management considerations of running wethers, hemi-castrates and induced cryptorchids for wool production', Australian Journal of Experimental Agriculture, v. 37, no. 3, pp. 303-310.

Gómez, MB, Castillo, M, Hick, MVH, Castillo, MF \& Frank, EN 2017, 'Revalorización de las aptitudes laneras de los biotipos ovinos de la región central argentina', Archivos de Zootecnia, v. 66, no. 255, 357-361.

Gordon, HMcL. \& Whitlock, HV 1939, 'A new technique for counting nematode eggs in sheep feces', Journal of the Council for Scientific and Industrial Research, v. 12, no. 1, pp. 50-52. 
Hopkins, DL, Jackson, RB \& Roberts, AHK 1992, 'Comparison of a modified cryptorchid treatment and castration: effect on growth, wool production, posthitis, testosterone production and development of masculine characteristics', Australian Journal of Experimental Agriculture, v. 32, no. 4, pp. 443-446.

Instituto Brasileiro de Geografia e Estatística2016,Banco de Dados: 2016, acessado 03 Outubro 2016, http://www.ibge.gov.br/

Khan, MJ, Abbas, A, Ayaz, M, Naeem, M, Akhter, MS \& Soomro, MH 2012 , 'Factors affecting wool quality and quantity in sheep', African Journal of Biotechnology, v. 11, no. 73, pp. 13761-13766.

Minola, J \& Elissondo, A 1990, Praderas y lanares - Tecnologia ovina sudamericana. Editora Hemisfério Sur. Buenos Aires. v. 1, 64p.

Oliveira, NRM \& Figueiró, PRP 1979, 'Efeitos da época de parição sobre a produção de lã, peso corporal de ovelhas e desenvolvimento de cordeiros da raça Corriedale', Ciência Rural, v. 9, no. 3, pp. 257-264.

Osório, JCS, Osório, MTM \& Sobrinho, AGS 2008, 'Morfologia e avaliação de carcaças ovinas'. In: Sobrinho, AGS, Sañudo, C, Osório, JCS, Arribas, MMC\& Osório, MTM, Produção de carne ovina: Técnicas de avaliação "in vivo" e na carcaça, $1^{a}$ ed, Funep, Jaboticabal. 228p.

Osório, JCS, Oliveira, NM, Osório, MTM, Jardim, RD \& Pimentel, MA 2002, 'Produção de carne em cordeiros cruza Border Leicester com ovelhas Corriedale e Ideal', Revista Brasileira de Zootecnia, v. 31, no. 3, pp. 1469-1480.

Osório, JCS, Osório, MTM, Vargas Junior, FM \& Leão, AG 2014, 'Produção e qualidade de lã', In: Selaive, AB \& Osório, JCS, Produção de ovinos no Brasil, $1^{a}$ ed, Roca, São Paulo. 656p.

Peña, S, Sacchero, D, Maurino, J, Lópes, GA, Abbiati, NN, Género, ER \& Martínez, RD 2016, 'Caracterización de la lana de ovejas Criollas argentinas em cuatro ambientes diferentes', Archivos de Zootecnia, v. 66 , no. $255,357-361$.

Sacchero, D, Willems, P \& Mueller, JP 2010, 'Perfiles de diámetro de fibra en lanas preparto de ovejas merino. 1. Estudio comparativo de líneas genéticas. Revista Argentina de Producción Animal, v. 30, no. 2, pp. 31-42.

Turner, AS \& Mcilwraith, CW 2002, Técnicas cirúrgicas em animais de grande porte, $1^{a}$ ed, Roca, São Paulo. 341p.

Viana, JGA \& Silveira, VCP 2009, 'Análise econômica da ovinocultura na metade sul do Rio Grande do Sul, Brasil', Ciência Rural, vol. 39, no. 4, pp. 1187-1192.

Wallace, ALC 2000,'The effect of hormones on wool growth', University of New England Publishing Unit. Armidale. pp. 257-268. 